\title{
Relajación secundaria en sistemas formadores de vidrios
}

\author{
D. GÓMEZ, A. ALEGríA Y J. COLMENERO \\ Dpto de Física de Materiales, Facultad de Química, Universidad del País Vasco, San Sebastián
}

En el trabajo que se presenta, se ha medido y analizado la relajación $\beta$ o secundaria en 15 sistemas formadores de vidrios (13 polímeros y 2 líquidos de bajo peso molecular) mediante Espectroscopía Dieléctrica de Banda Ancha entre $10^{-2} \mathrm{~Hz}$ y $10^{7} \mathrm{~Hz}$ a temperaturas inferiores y ligeramente superiores a la de transición vítrea. En el análisis de los resultados experimentales se ha considerado la relajación $\beta$ como un proceso activado térmicamente con una distribución gaussiana de energías de activación. Los resultados obtenidos de este modo han sido comparados con recientes teorías que relacionan este fenómeno con el de la transición vítrea.

Palabras clave: Relajación secundaria, espectroscopía dieléctrica, sistemas formadores de vidrios, transición vitrea, energía de activación.

\section{Secondary Relaxation in glass forming systems}

In this work, the $\beta$ or secondary relaxation of 15 glass forming systems (13 polymers and 2 low molecular weight liquids) has been analysed by means of Broadband Dielectric Spectroscopy from $10^{-2} \mathrm{~Hz}$ to $10^{7} \mathrm{~Hz}$ at temperatures below and slightly above the glass transition temperature. In further analysis of the experimental results, it has been considered this relaxation as an thermally activated process with a gaussian distribution of activation energies. The results obtained in this way have been compared with recent theories that relate this phenomenology with the glass transition one.

Key words: Secondary relaxation, dielectric spectroscopy, glass forming systems, glass transition, activation energy.

\section{INTRODUCCION}

En el estudio de la dinámica de sistemas formadores de vidrios, tradicionalmente, se ha puesto gran interés en el conocimiento de la relajación primaria o relajación $\alpha$. Así, se ha asociado ésta al fenómeno de la transición vítrea, relacionada con el movimiento cooperativo de las moléculas en el material. De modo abreviado, podemos decir que esta relajación viene caracterizada por una función de relajación exponencial extendida $\left(\phi(t)=\exp \left(-(t / \tau)^{\beta}\right)\right)$ y una dependencia del tiempo de relajación característico, $\tau$, con la temperatura de acuerdo con la ley de Vogel-Fulcher $\left[\tau=\tau_{0} \exp \left(\mathrm{DT}_{0} /\left(\mathrm{T}-\mathrm{T}_{0}\right)\right)\right]$. En general, la temperatura de transición vítrea $\left(\mathrm{T}_{\mathrm{g}}\right)$ se define como aquella a la que el tiempo de relajación toma un cierto valor, típicamente del orden de 100 segundos, dada la inaccesibilidad experimental de la temperatura de transición vítrea ideal $\left(\mathrm{T}_{0}\right)$.

Sin embargo, por debajo de esta temperatura (estado vítreo) no cesan todos los movimientos moleculares, evidenciándose pequeños movimientos a escala molecular que permanecen. En 1970, Johari y Goldstein (1) detectan la presencia de una relajación secundaria, o relajación $\beta$, que aparece en la mayoría de los sistemas formadores de vidrios, tanto a temperaturas menores como mayores que $\mathrm{T}_{\mathrm{g}}$. Posteriormente, se ha observado que esta relajación juega un papel importante en las propiedades de estos materiales (2) y podría permitir establecer una relación entre propiedades térmicas de baja temperaturas y la transición vítrea. Las principales características fenomenológicas de este proceso son una función de relajación muy extendida y un comportamiento tipo Arrhenius $\left[\tau=\tau_{0} \exp \left(E_{\text {act }} / \mathrm{RT}\right)\right]$ del tiempo de relajación característico, donde $\mathrm{E}_{\text {act }}$ representa la energía de activación de un proceso simple activado térmicamente. El origen físico de este proceso es controvertido y sigue siendo objeto de debate en nuestros días. Por un lado, algunos autores (3) apoyan la idea inicialmente propuesta por Johari y Goldstein, que consideran el origen de esta relajación en estados configuracionales del líquido subenfriado que no se alteran durante la formación del vidrio. Estos "defectos" en la estructura se han dado en llamar "islas de movilidad" en la literatura. Según esta descripción, la relajación secundaria tiene el mismo origen intermolecular que la relajación $\alpha$ y es, por tanto, una característica intrínseca del estado vítreo. Por otro lado, se ha tratado de explicar este fenómeno desde el punto de vista intramolecular, relacionándolo con movimientos de subgrupos moleculares (4). En sistemas poliméricos esta relajación es a menudo interpretada como movimientos rotacionales de grupos unidos a la cadena principal o mecanismos tipo "manivela" en la misma.

En el presente trabajo, se ha realizado un estudio de diferentes sistemas formadores de vidrios (13 polímeros y 2 líquidos de bajo peso molecular) mediante Espectroscopía Dieléctrica y se han analizado los resultados de acuerdo a la interpretación comúnmente aceptada para la relajación secundaria como un proceso activado térmicamente con una distribución gausiana de energías de activación (5). Los resultados obtenidos mediante este análisis se discuten teniendo en cuenta las teorías expuestas en el párrafo anterior. 


\section{PROCEDIMIENTO EXPERIMENTAL}

Las medidas de la constante dieléctrica en función de la frecuencia se han llevado a cabo entre $10^{-2} \mathrm{~Hz}$ y $10^{7} \mathrm{~Hz}$ con un sistema de control de temperatura Novocontrol y un analizador Solartron-Schlumberger SI1260 unido a un convertidor BDC. El error experimental en la determinación de la tangente de pérdidas ( $\left.\tan \delta=\varepsilon^{\prime \prime} / \varepsilon^{\prime}\right)$ es $10^{-4}$. Las medidas se han realizado de modo isotermo, cada $10 \mathrm{~K}$, siendo el intervalo de temperaturas medido entre $\mathrm{T}_{\mathrm{g}}-150 \mathrm{~K}$ y $\mathrm{T}_{\mathrm{g}}+30 \mathrm{~K}$, aproximadamente, y la incertidumbre en la determinación de la temperatura menor de $0.15 \mathrm{~K}$. Las muestras poliméricas fueron adquiridas comercialmente y preparadas sobre la superficie de los electrodos a partir de una disolución de tetrahidrofurano y por evaporación del disolvente. Los líquidos de bajo peso molecular fueron depositados directamente sobre los electrodos. En ambos casos se mantuvo la distancia entre electrodos constante mediante la inserción de separadores de Teflon (PTFE) de $0.1 \mathrm{~mm}$ de espesor, dado que este material es inactivo dieléctricamente en el rango de frecuencias medido.

\section{RESULTADOS Y ANÁLISIS}

En la Figura 1 se han representado, a modo de ejemplo, los resultados obtenidos para la parte imaginaria de la constante dieléctrica de algunos de los compuestos estudiados. Se han elegido temperaturas a las cuales el máximo de pérdidas se encuentra en torno al centro de la ventana experimental de medida. Como puede verse, la amplitud es, en general, pequeña respecto a los valores típicos correspondiente a la relajación $\alpha$ y su anchura es todos los casos grande.

El análisis de los resultados experimentales se ha realizado suponiendo que la forma espectral de la relajación $\beta$ puede ser descrita por una función gaussiana en el logaritmo de la frecuencia. Además, se ha sumado un fondo constante, relacionado con la zona de alta frecuencia correspondiente a la relajación $\alpha$ o la contribución correspondiente a la parte de alta frecuencia de la relajación $\alpha$, cuando ésta y el proceso $\beta$ se encuentran cercanos. La contribución de la relajación $\beta$ viene descrita por

$$
\varepsilon^{\prime \prime}(v)=\frac{\Delta \varepsilon}{\sqrt{2 \pi} \sigma_{e x p}} \exp \left[-\frac{\left(\log v-\log v_{p}\right)^{2}}{2 \sigma_{e x p}^{2}}\right]
$$

siendo $\sigma_{\text {exp }}$ la anchura y $v_{p}$ la frecuencia del máximo. Esta descripción esta de acuerdo con la hipótesis comúnmente aceptada según la cual existen mínimos locales de energía separados por barreras de potencial. Así, la dinámica de estos movimientos involucra distribuciones anchas de tiempos de relajación, que teniendo en cuenta el comportamiento Arrhenius de la relajación puede ser interpretado como una distribución normal de energías de activación

$$
g_{\beta}(E)=\frac{1}{\sqrt{2 \pi} \sigma_{E}} \exp \left[-\frac{\left(E-E_{a c t}\right)^{2}}{2 \sigma_{E}^{2}}\right]
$$

siendo $\mathrm{E}_{\text {act }}$ el valor medio de la energía de activación y $\sigma_{\mathrm{E}} \mathrm{su}$ anchura.

De este modo, es posible mediante [1] obtener los valores de la energía de activación media para los compuestos estudiados ajustando los valores de $v_{p}$ a la ecuación de Arrhenius $\left(v_{\mathrm{p}}=v_{0} \exp \left(-\mathrm{E}_{\mathrm{act}} / \mathrm{kT}\right)\right)$. Este ajuste resulta satisfactorio en todos los casos.

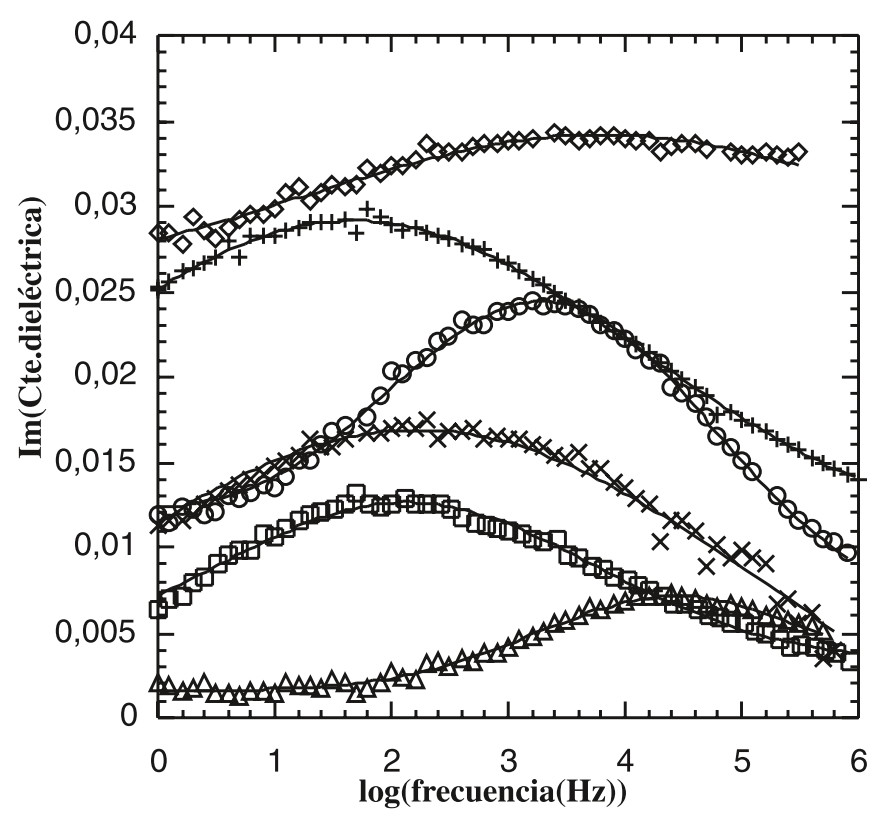

Figura 1: Resultados obtenidos para la parte imaginaria de la constante dieléctrica de algunos de los compuestos estudiados: dietil-ftalato (DEP) (O) (155K), tetrametil-bisfenol-policarbonato (TMBPC) $(\square)(350 \mathrm{~K})$, polietileno-acrilato (PEA) $(\diamond)$ $(140 \mathrm{~K})$, polivinil- metil-eter (PVME) $(\mathrm{X})(118 \mathrm{~K})$, fenoxi $(\mathrm{PH})$ $(+)(175 \mathrm{~K})$ y polisulfona $(\mathrm{PSF})(\triangle)(240 \mathrm{~K})$. Las líneas continuas corresponden a los ajustes realizados de acuerdo con el texto.

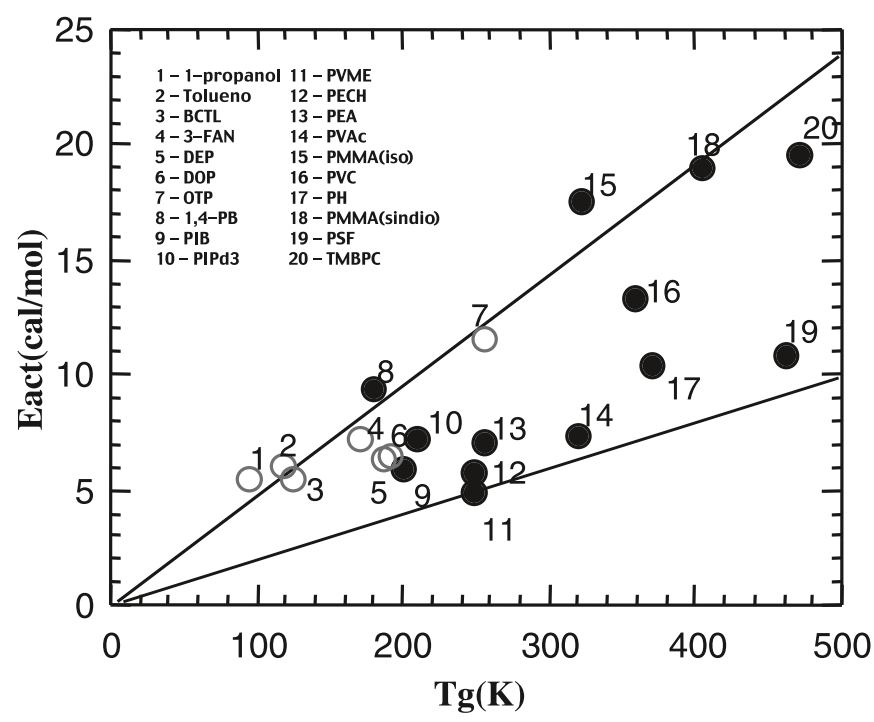

Figura 2. Energías de activación obtenidas para sistemas de bajo peso molecular $(\bigcirc)$ y sistemas poliméricos $(\bullet)$ en función de la temperatura de transición vítrea. Los valores correspondientes al 1-propanol y tolueno han sido tomados de (3), el de cloro benzil tolueno de (4), el de 3-fluor anilina de (7) y el de OTP de (1). Las líneas continuas representan $\mathrm{N}=10$ (6) y $\mathrm{N}=24$ (3). 
También es posible obtener la anchura de la función $g_{\beta}(E)$, $\sigma_{E^{\prime}}$ a partir de $\sigma_{\text {exp }}$ a través de la anchura correspondiente a una distribución gaussiana de tiempos de relajación, $\sigma_{\log \tau}$. Puede verse que

$$
\begin{aligned}
& \sigma_{\log \tau}=-0.4118+1.2291 \sigma_{\exp }-0.03641 \sigma_{\exp }^{2} \\
& \sigma_{\mathrm{E}}=\sigma_{\log \tau} \mathrm{kT} \operatorname{Ln} 10
\end{aligned}
$$

Los valores de $\sigma_{\mathrm{E}}$ obtenidos muestran un suave descenso con la temperatura para todos los compuestos.

\section{4.- DISCUSION Y CONCLUSIONES}

En la Figura 2 se muestran los valores de la energía de activación en función de la temperatura de transición vítrea para los compuestos estudiados y otros obtenidos de la literatura. Esta representación es interesante a fin de comprobar la validez de la ley $\mathrm{E}_{\text {act }}=\mathrm{NRT}_{\mathrm{g}}$ propuesta recientemente por algunos autores que intentan relacionar la relajación secundaria con el fenómeno de la transición vítrea $(3,6)$. Puede observarse que:

(a) Existe una cierta correlación entre ambas magnitudes, aunque no es posible obtener una ley bien definida. El parámetro N varía desde 10.12 para el PVME hasta 29.17 para el 1-propanol.

(b) Si comparamos los valores obtenidos para polímeros y para líquidos de bajo peso molecular se observa una dependencia más fuerte ( $\mathrm{N}$ mayor) y definida ( $\Delta \mathrm{N}$ menor) en estos últimos, pudiéndose plantear la posibilidad de diferencias en los mecanismos de relajación entre ambos tipos de materiales.

\section{AGRADECIMIENTOS}

Este trabajo ha sido financiado por el Ministerio de Educación y Cultura (MEC) (proyecto PB97-0638), Gobierno Vasco (proyecto GV-PI/1998/20) y Universidad del País Vasco (proyectos 206.215-G20/98). Los autores agradecen igualmente la financiación parcial de la Diputación Foral de Guipúzcoa e Iberdrola S.A. D.G. agradece la beca predoctoral otorgada por el MEC.

\section{BIBLIOGRAFÍA}

1. G.P. Johari, M. Goldstein. "Viscous Liquids and the glass transition. III. Secondary Relaxations in aliphatic alcohols and other nonrigid molecules". J. Chem. Phys. 55, 9, 4245-4252 (1971)

2. N.O. Birge, Y.H. Jeong, S.R. Nagel, S. Bhattacharya, S. Susman. “Distribution of relaxation times in $(\mathrm{KBr})_{0.5}(\mathrm{KCN})_{0.5}$ ". Phys. Rev. B 30, 2306 (1984).

3. A. Kudlik, C. Tschirwitz, S. Benkhof, T. Blochowicz, E. Rössler. “Slow secondary process in supercooled liquids" Europhys. Lett. 40, 6, 649 (1997).

4. L. Wu. "Relaxation mechanisms in a benzyl chloride-toluene glass"Phys. Rev. B 43, 12, 9906 (1991)

5. R.D. Deegan, S.R. Nagel. “Dielectric susceptibility measurements of the primary and secondary relaxation in polybutadiene"Phys. Rev. B 52, 5653 (1995).

6. B. Frick, D. Richter. "The microscopic basis of the glass transition in polymers from neutron scattering studies" Science 267, 1939 (1995).

7. A. Kudlik, C. Tschirwitz, T. Blochowicz, S. Benkhof, E. Rössler. "Slow secondary relaxation in simple glass formers" J. Non-Crys. Sds. 235-237, 406-411 (1998). 